\title{
Evolution of Solitary and Group Transport Behaviors for Autonomous Robots Capable of Self-Assembling
}

\author{
Roderich Groß, ${ }^{1,2}$ Marco Dorigo ${ }^{2}$ \\ ${ }^{1}$ ANT LAB, School of Biological Sciences, University of Bristol, UK \\ ${ }^{2}$ IRIDIA, CoDE, Université Libre de Bruxelles, Belgium
}

\begin{abstract}
Group transport is performed in many natural systems and has become a canonical task for studying cooperation in robotics. We simulate a system of simple, insect-like robots that can move autonomously and grasp objects as well as each other. We use artificial evolution to produce solitary transport and group transport behaviors. We show that robots, even though not aware of each other, can be effective in group transport. Group transport can even be performed by robots that behave as in solitary transport. Still, robots engaged in group transport can benefit from behaving differently from robots engaged in solitary transport. The best group transport behaviors yielded by half of the evolutions let robots organize into self-assembled structures. This provides evidence that self-assembly can provide adaptive value to individuals that compete in an artificial evolution based on task performance. We conclude the article by discussing potential implications for evolutionary biology and robotics.
\end{abstract}

Keywords group transport - solitary - social behavior - evolution of cooperation - self-assembly . autonomous robots $\cdot$ evolutionary robotics $\cdot$ swarm robotics $\cdot$ swarm intelligence . evolutionary biology

\section{Introduction}

Group transport can be defined as the "conveyance of a burden by two or more individuals" (Moffett, 1992, p. 227). Group transport is performed in many natural systems (Franks, 1986; Klumpp \& Lipowsky, 2005; Moffett, 1992; Vakanas \& Krafft, 2004). It has also become a canonical task for studying cooperation in artificial systems (Gerkey \& Matarić, 2002; Kosuge \& Oosumi, 1996; Kube \& Zhang, 1993; Parker, 1999; Sugar \& Kumar, 2002). When compared to solitary transport, it offers the advantage of being more reliable and in addition more powerful, as a group may exert higher forces onto an object than each of the participating individuals alone.

Correspondence to: Roderich Groß, Ecole Polytechnique Fédérale de Laussanne, EPFL-STI-IMT-LSRO, Station 9, CH-1015 Lausanne, Switzerland. E-mail: roderich.gross@ieee.org Tel: +41216937844
We use evolutionary algorithms to produce solitary transport and group transport behaviors for a system of simulated robots (Harvey, Husbands, Cliff, Thompson, \& Jakobi, 1997; Nolfi \& Floreano, 2000). Similar to many animals, our robots can move based on their own propulsion and have a mechanism by which they can grasp an object to be transported or another robot. Their cognitive capabilities are very limited and similar to those of solitary animals: they can neither perceive teammates nor communicate with them directly. The task is to move the object in an arbitrary direction.

The aim of the study presented in this article is twofold. First, we want to understand to what extent individuals, which as a group have to accomplish a cooperative task, can benefit from behaving differ-

Copyright $(92008$ International Society for Adaptive Behavior (2008), Vol 16(5): 285-305.

DOI: $10.1177 / 1059712308090537$ 
ently from individuals that have to accomplish tasks on their own. In particular, we examine whether individuals engaged in group transport can benefit from behaving differently from individuals engaged in solitary transport. Answers to this research question are relevant for the design of robotic systems. A deeper understanding of the relation between solitary and social behavior can also shed light on the evolution of cooperation in animal groups and societies, which is one of the most important unanswered questions in evolutionary biology.

Second, we examine whether evolutionary algorithms can yield behaviors that let individuals organize into physically connected pushing or pulling structures to accomplish a task. This "self-assembly" ability, without being explicitly favored by the fitness function of the evolutionary algorithm, can evolve if providing an adaptive value for the individuals. This can lead to new insight for the design of robotic systems. Moreover, gaining some basic understanding of the factors that favor self-assemblage formation is one focus of current research in biology (Anderson, Theraulaz, \& Deneubourg, 2002; Theraulaz et al., 2001).

Group transport and self-assembly are both widely observed phenomena in ant colonies (Anderson et al., 2002; Franks, 1986; Moffett, 1992). In both cases, ants temporarily organize into functional units at a level intermediate to that of the individual and that of the colony (Anderson \& McShea, 2001). In designing group transport systems, we follow some basic principles that are used to explain the behavior of such functional units, the behavior of whole colonies, and collective animal behavior in general (Camazine et al., 2001; Garnier, Gautrais, \& Theraulaz, 2007; Grassé, 1959; Sumpter, 2006; Theraulaz \& Bonabeau, 1999). We emphasize system properties, such as the following:

1. Decentralization, that is, robots follow rules in a fully autonomous and distributed manner.

2. Locality of perception/indirect communication, that is, robots perceive objects in a limited range only, and communicate through physical interactions with their environment.

3. Redundancy, that is, the robotic system can continue to function even when faced with a moderate reduction in its workforce.

Evolutionary algorithms (and other populationbased metaheuristic optimization algorithms) have already proven successful in reproducing, with (simulated or real) autonomous robots, several collective capabilities known from social insects and other animals, including aggregation (Dorigo et al., 2004; Pugh \& Martinoli, 2006), flocking or schooling (Baldassarre, Nolfi, \& Parisi, 2003; Quinn, Smith, Mayley, \& Husbands, 2003; Reynolds, 1993; Spector, Klein, Perry, \& Feinstein, 2005; Ward, Gobet, \& Kendall, 2001), foraging (Panait \& Luke, 2003; Peréz-Uribe, Floreano, \& Keller, 2003), and inspection/patrolling (Martinoli, 1999; Zhang, Antonsson, \& Martinoli, 2006). Recently, Peréz-Uribe et al. (2003) and Floreano, Mitri, Magnenat, and Keller (2007) studied evolutionary conditions for the "emergence" of cooperative behavior in groups of robots that perform a foraging task. They found that cooperation (in the form of information transfer using pre-existing communication devices) evolved best when groups consisted of genetically identical individuals and when selection acted at the level of groups.

In contrast to other work in the literature, we study the evolution of cooperation by investigating the relation between solitary behavior and social behavior. We do so by focusing on relatively primitive forms of cooperation that are likely to be available when solitary individuals encounter each other. We use a physicsbased three-dimensional simulator, in which robots can influence each other by means of physical interaction, both directly and indirectly (i.e., through the object being manipulated). Coordination can also be implicit, for instance, when the behaviors of the robots exploit invariants or cues present in the environment.

In the evolutionary algorithm used, a "genotype" encodes a simple recurrent neural network, which is cloned and copied to each robot within a group. Thus, all members of a group are genetically identical. However, this does not preclude variability in their behaviors, as each of the robots in a group can be different, in terms of its phenotype ${ }^{1}$ and of the experience gained during its lifetime. We consider a population of genotypes (i.e., a population of groups). Selection acts at the level of genes (Dawkins, 1976; Hamilton, 1964).

We consider two types of environments. The first is used to evolve solitary transport behaviors. Only one robot and one light object are present, and consequently behavior is not selected for being social. The second type of environment is used to evolve group transport behaviors. In this setup, two robots and a heavy object are present, and the object cannot be 

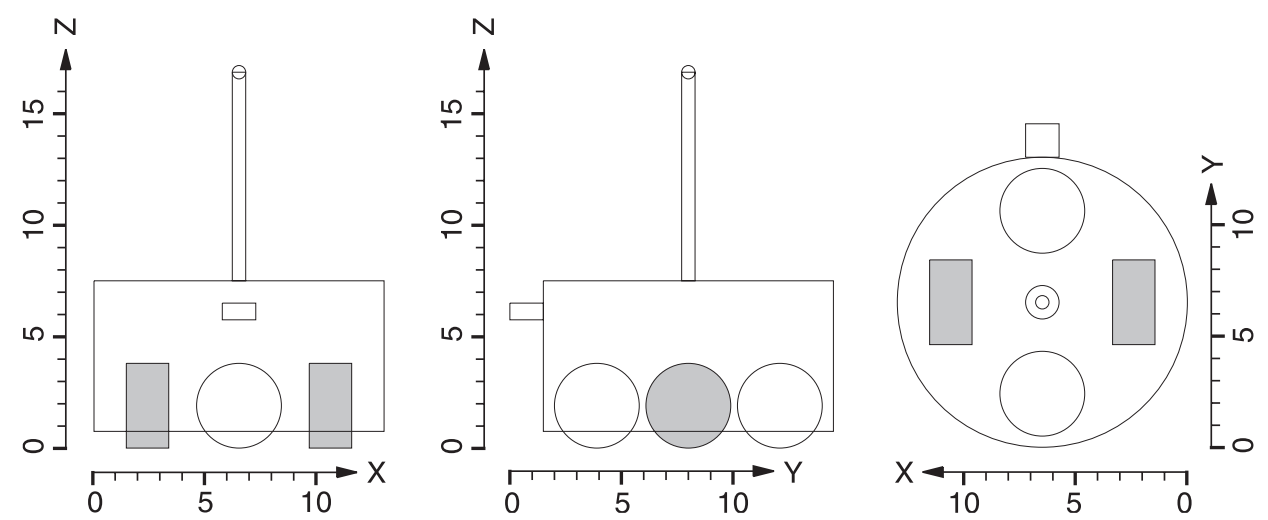

Figure 1 The simulation model of the robot: front, side, and top view (units in $\mathrm{cm}$ ).

moved without cooperation. Any behavior must be social, either mutually beneficial or spiteful (West, Gardner, \& Griffin, 2006). Note that, in this setup, selection at the level of genes is equivalent to betweengroup selection (Bourke \& Franks, 1995; Foster, Wenseleers, \& Ratnieks, 2006; Korb \& Heinze, 2004).

The article is organized as follows. In Section 2, we detail the methods, and in Section 3 we describe the results of our study on the evolution of solitary and group transport behavior. In Section 4 we overview the related work, and in Section 5 we discuss the results and conclude the article.

\section{Methods}

In the following, we detail the task, the simulation model, the robot's controller, and the evolutionary algorithm.

\subsection{Task}

We study solitary and group transport of an object, hereafter also called the "prey." The robots' environment consists of a flat ground, the prey, and a light source. The prey is modeled as a cylinder, $10 \mathrm{~cm}$ in height and $12 \mathrm{~cm}$ in radius. For solitary transport (i.e., one robot), we use a prey of mass $250 \mathrm{~g}$; for group transport (i.e., two robots), we use a prey of mass $500 \mathrm{~g}$. The $500-\mathrm{g}$ prey cannot be moved by a single robot. The light source represents an environmental cue and as such can be exploited by the robots to coordinate their actions. Initially, the robots are put at ran- dom positions near the prey. The task is to move the prey in an arbitrary direction (the farther the better).

\subsection{Simulation Model}

The simulator models the kinematics and dynamics of rigid, partially constrained, bodies in three dimensions using the Vortex ${ }^{\mathrm{TM}}$ simulation toolkit (CMLabs Simulations, Inc., Canada). Frictional forces are calculated based on the Coulomb friction law (Coulomb, 2001). The model of the robot is illustrated in Figure 1. It is an approximation of a physical robot, called s-bot, which was designed and implemented in the context of the Swarm-bots project (Dorigo, 2005; Dorigo et al., 2006; Mondada et al., 2005). The model is composed of five bodies: two spherical wheels, two cylindrical wheels, and a cylindrical torso. The torso is composed of several parts that are rigidly linked: a cylindrical body, a protruding cuboid (in what we define to be the robot's front), and a pillar fixed on top. The spherical wheels are linked to the chassis via ball-and-socket joints. The cylindrical wheels are linked to the chassis via hinge joints. Each wheel weighs $20 \mathrm{~g}$. The robot has a total mass of $660 \mathrm{~g}$.

The robot's actuators and sensors are summarized in Table 1. The cylindrical wheels are motorized, and can be moved with angular speeds $w_{\mathrm{L}}$ and $w_{\mathrm{R}}$ (in rad s $\mathrm{r}^{-1}$ ) in the range $[-M, M](M \approx 15)$. The cuboid heading forward represents a connection device (e.g., a gripper). If it is in contact with either the cylindrical body of another robot or the (cylindrical) prey, a physical connection can be established ( $c=1$, and $c=0$ otherwise). Connections can be released at any time. In par- 
Table 1 Summary of the robot's actuators and sensors. Units are in $\mathrm{cm}$, rad, and $\mathrm{rad} \mathrm{s}^{-1}$. See text for details.

\begin{tabular}{ll} 
Actuators & \\
Left wheel (angular speed) & $w_{\mathrm{L}} \in[-M, M]$ \\
Right wheel (angular speed) & $w_{\mathrm{R}} \in[-M, M]$ \\
Connection mechanism & $c \in\{0,1\}$ \\
\hline Sensors (exteroceptive) & \\
Light source (angular position) & $\alpha \in[0,2 \pi]$ \\
Prey (angular position) & $\beta \in[0,2 \pi]$ \\
Prey (distance) & $d \in[0, R]$ \\
\hline Sensors (proprioceptive) & \\
Connection mechanism & $c^{\prime} \in\{0,1\}$ \\
\hline
\end{tabular}

ticular, this will occur if the intensity of the force transmitted by the connection mechanism exceeds a certain threshold. As a consequence, it is not possible for the robots to form very long pulling chains. The robot is equipped with an omni-directional camera mounted on a pillar support, which is fixed at the center of the torso's top. The camera is able to detect the angular position $\alpha$ of the light source. Moreover, it provides the angular position $\beta$ and distance $d$ of the prey, if the latter resides within the sensing range $(R \approx$ $50 \mathrm{~cm}$ ). In simulation, angles and distances can be calculated from the positions of other objects in the scene. ${ }^{2}$ A connection sensor enables a robot to perceive whether it is connected to another object $\left(c^{\prime}=1\right)$ or not $\left(c^{\prime}=0\right)$. The robot is not equipped with any sensor capable of detecting the presence of a teammate.

Random noise affects the characteristics of the robot's actuators and sensors (i.e., variables $w_{\mathrm{L}}, w_{\mathrm{R}}$, $M, \alpha, \beta, d$, and $R$ ). We model two different types of random noise, as follows:

1. Random variables $\xi_{1}, \xi_{2}, \xi_{3}, \ldots$ generated for each robot only once at the beginning of its lifetimethey model differences among the hardware of the robots.

2. Random variables $\xi_{1}^{t}, \xi_{2}^{t}, \xi_{3}^{t}, \ldots$ generated for each robot at each time step $t$ during its lifetimethey model temporary fluctuations in the behavior of the robot's actuators and sensors.

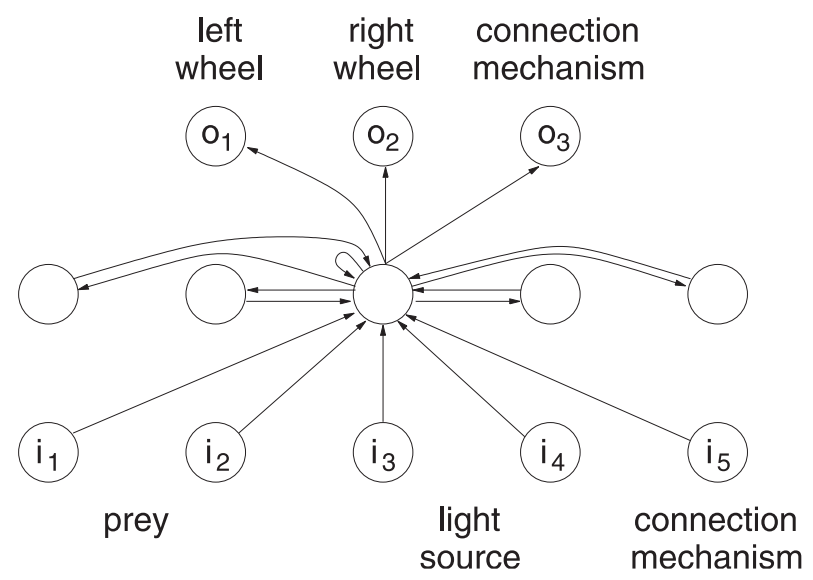

Figure 2 The neural network controller comprising five input neurons (bottom), five hidden neurons (center), and three output neurons (top). Only the synaptic connections to and from the neuron in the center of the hidden layer are illustrated. An additional bias neuron (not shown), providing a constant input of 1 , is connected to each neuron of the hidden layer and the output layer.

Let $N\left(\mu, \sigma^{2}\right)$ denote the normal distribution with mean $\mu$ and variance $\sigma^{2}$. The value $w_{\mathrm{L}}$ (in rad s $\mathrm{s}^{-1}$ ) is initially within the range $[-15,15]$ and modified by multiplication with $\xi_{1} \xi_{1}^{t}$, where $\xi_{1}$ follows $N\left(1,0.02^{2}\right)$ and $\xi_{1}^{t}$ follows $N\left(1,0.05^{2}\right)$. If $w_{\mathrm{L}}$ is less than $\xi_{2}$, which follows the uniform distribution $U(0.1,0.5)$, then the speed is set to zero. The value $w_{\mathrm{R}}$ is modified in a similar way using independent random variables $\xi_{3}, \xi_{3}^{t}$, and $\xi_{4}$. The camera is assumed to be calibrated with no bias in the error. The only exception to this is the sensing range $R$ (in $\mathrm{cm}$ ) that is set to $\xi_{5}$, which follows $N(50,1)$. Angle $\alpha$ (in rad) is modified by adding $\xi_{6}^{t}$, which follows $N\left(0,0.1^{2}\right)$, and angle $\beta$ is modified by adding $\xi_{7}^{t}$, which follows $N\left(0,0.1^{2}\right)$. Distance $d$ (in $\mathrm{cm})$ is modified by adding $\xi_{8}^{t}$, which follows $N(0,1)$.

\subsection{Controller}

All the robots of a group are initially assigned an identical controller. Every $100 \mathrm{~ms}$, a control loop spreads activation in a neural network taking input from the robot's sensors, and uses the outputs as motor commands. The neural network is illustrated in Figure 2. It is a simple recurrent neural network (Elman, 1990) and has an input layer of five neurons $\left(i_{1}, i_{2}, i_{3}, i_{4}\right.$, and $i_{5}$ ), a hidden layer of five (fully interconnected) neurons, and an output layer of three neurons $\left(o_{1}, o_{2}\right.$, and 
$o_{3}$ ). The weights of the synaptic connections of the network are genetically encoded parameters. The activations of the hidden and output neurons are mapped into the range $(0,1)$ using the sigmoid function

$$
f(x)=\frac{1}{1+\exp (-x)} .
$$

The activations of the five input neurons are computed based on the robot's sensor readings (see Table 1):

$$
\begin{aligned}
& i_{1}= \begin{cases}\left(1-\frac{d}{R}\right) \sin \beta & \text { if } d<R \\
0 & \text { otherwise }\end{cases} \\
& i_{2}= \begin{cases}\left(1-\frac{d}{R}\right) \cos \beta & \text { if } d<R \\
0 & \text { otherwise }\end{cases} \\
& i_{3}=\sin \alpha, \\
& i_{4}=\cos \alpha, \\
& i_{5}=c^{\prime}
\end{aligned}
$$

The activations of the three output neurons are used to set the motor commands (see Table 1):

$$
\begin{aligned}
& w_{\mathrm{L}}=M\left(2 o_{1}-1\right), \\
& w_{\mathrm{R}}=M\left(2 o_{2}-1\right), \\
& c= \begin{cases}0 & o_{3}<0.5 ; \\
1 & \text { otherwise. }\end{cases}
\end{aligned}
$$

\subsection{Evolutionary Algorithm}

The evolutionary algorithm used is a self-adaptive version of $\mathrm{a}(\mu+\lambda)$ evolution strategy (Beyer, 2001; Schwefel, 1975). Each individual ${ }^{3}$ is composed of $n=73$ real-valued object parameters $x_{1}, x_{2}, \ldots, x_{n}$ specifying the connection weights of the neural network controller, and the same number of real-valued strategy parameters $s_{1}, s_{2}, \ldots, s_{n}$ specifying the mutation strength used for each of the $n$ object parameters.
The initial population of $\mu+\lambda$ individuals is constructed randomly. In each generation, all individuals are assigned a fitness value. The best-rated $\mu$ individuals are selected to create $\lambda$ offspring. Subsequently, the $\mu$ parent individuals and the $\lambda$ offspring are copied into the population of the next generation. Note that the $\mu$ parent individuals that are replicated from the previous generation are re-evaluated. We have chosen $\mu=20$ and $\lambda=80$.

Each offspring is created by recombination with probability 0.2 and by mutation otherwise. In either case, the parent individual(s) is selected randomly. As recombination operators, we use intermediate and dominant recombination (Beyer, 2001), both with the same probability. The offspring is subjected to mutation. The mutation operator changes the object parameter $x_{i}$ by adding a random variable $\xi_{a}$, which follows the normal distribution $N\left(0, s_{i}^{2}\right)$ :

$$
x_{i}=x_{i}+\xi_{a}
$$

Prior to the mutation of object parameter $x_{i}$, the "mutation strength" parameter $s_{i}$ is multiplied by a random variable that follows a lognormal distribution (Beyer, 2001; Schwefel, 1975):

$$
s_{i}=s_{i} \exp \left(\xi_{g}+\xi_{s}\right),
$$

where $\xi_{g}$, which is generated once for all strategy parameters, follows the normal distribution $N\left(0, \tau_{g}^{2}\right)$, and $\xi_{s}$, which is generated for each of the strategy parameters $s_{1}, s_{2}, s_{3}, \ldots$, follows the normal distribution $N\left(0, \tau_{s}^{2}\right) . \tau_{g}$ and $\tau_{s}$ are commonly set to $1 / \sqrt{2 n}$ and $1 / \sqrt{2 \sqrt{n}}$, respectively (see Yao, Liu, \& Lin, 1999 and references therein). To prevent premature convergence, a lower bound of 0.01 for the strategy parameters is applied (Liang, Yao, Liu, Newton, \& Hoffman, 1998).

2.4.1 Fitness Computation The fitness of individuals is assessed using simulations. Each trial lasts $T=$ 20 simulated seconds. Initially, the prey is placed in what we refer to as the center of the environment. The light source is placed at a random position $300 \mathrm{~cm}$ away from the prey. This is less than the distance the prey can be moved within the simulation time $T . N \in$ $\{1,2\}$ robots are placed at random positions and orientations, but not more than $R / 2=25 \mathrm{~cm}$ away from 
the perimeter of the prey. This ensures that the prey can initially be detected by each robot.

The measure of quality $\mathcal{Q}$ accounts for the ability of the individual to let the robots remain in the vicinity of the prey, and transport it, the farther the better, in an arbitrary direction. It is defined as

$$
\mathcal{Q}= \begin{cases}\mathcal{C} & \text { if } \mathcal{T}=0 \\ 1+\left(1+\mathcal{T}^{\rho_{1}}\right) \mathcal{C}^{\rho_{2}} & \text { otherwise }\end{cases}
$$

where $\mathcal{C} \in[0,1]$ reflects the clustering performance, $\mathcal{T} \in[0, \infty)$ reflects the transport performance, and $\rho_{1}=$ 0.5 as well as $\rho_{2}=5$ are parameters that were determined by trial and error.

The clustering performance $\mathcal{C}$ is defined as

$$
\mathcal{C}=\frac{1}{N} \sum_{i=1}^{N} \mathcal{C}_{i}
$$

with

$$
\mathcal{C}_{i}= \begin{cases}0 & \text { if } d_{i}^{T}>R \\ 1 & \text { if } d_{i}^{T}<R / 2 \\ \frac{R-d_{i}^{T}}{R / 2} & \text { otherwise }\end{cases}
$$

where $d_{i}^{T}$ denotes the distance between robot $i$ and the perimeter of the prey at time $T$ (see Table 1). If the prey at time $T$ is not within the sensing range $R$ of a robot, the latter receives the lowest possible reward (i.e., 0). Robots that at the end of the trial are still within the initial range $(R / 2=25 \mathrm{~cm})$ around the prey receive the maximum reward (i.e., 1$)$. Note that $\mathcal{C}$ does not impose any bias on the transport strategy: any pulling or pushing arrangement of two robots is assigned the maximum clustering performance.

The transport performance $\mathcal{T}$ is defined as

$$
\mathcal{T}=\Delta\left(\mathcal{X}^{0}, \mathcal{X}^{T}\right),
$$

where $\mathcal{X}^{t}, t \in[0, T]$ denotes the position of the prey at time $t$, and $\Delta(\cdot, \cdot)$ is the Euclidean distance.

The performance of an individual is evaluated in $S=5$ independent trials. For each trial, the start con- figuration (e.g., specifying the initial locations of the robots and of the light source) is randomly generated. Every individual within the same generation is evaluated on the same sample of start configurations. The sample is changed once at the beginning of each generation. Let $\mathcal{Q}_{i}$ be the quality observed in trial $i$, and let $\phi$ be a permutation of $\{1,2, \ldots, S\}$ so that $\mathcal{Q}_{\phi(1)} \leq$ $\mathcal{Q}_{\phi(2)} \leq \ldots \leq \mathcal{Q}_{\phi(S)}$. Then the fitness $\mathcal{F}$, which is to be maximized, is defined as

$$
\mathcal{F}=\frac{2}{S(S+1)} \sum_{i=1}^{S}(S-i+1) \mathcal{Q}_{\phi(i)}
$$

Note that in this way the trial resulting in the lowest transport quality value (if any) has the highest impact on $\mathcal{F}$. Thereby, individuals are penalized for exhibiting high performance fluctuations.

\section{Results}

We conducted 30 independent evolutionary runs for 150 generations each. This corresponds to 15,000 fitness evaluations per run. This limit was defined in order to keep the execution time per run within a time frame of one to four days. In 20 runs, the fitness of individuals reflected the performance in solitary transport (i.e., simulations with a single robot and a prey of weight $250 \mathrm{~g}$ ), whereas in the other 10 runs, the fitness reflected the performance in group transport (i.e., simulations with two robots and a prey of weight $500 \mathrm{~g}$ ). ${ }^{4}$ Recall that the 500 -g prey cannot be moved by a single robot. Figures 3 and 4 present the corresponding average and maximum fitness time histories. The curves correspond respectively to the average of 20 and 10 runs with different random seeds. The values are normalized in the range $[0,1]$. The lower bound is tight, and represents trials in which the prey was not moved and the robots lost visual contact with it (fitness zero). The upper bound corresponds to the maximum distance a robot that is pre-assembled with the lighter ( $250 \mathrm{~g}$ ) prey can push the latter within $T=20 \mathrm{~s}$. To compute the upper bound, we disabled any random noise affecting the actuators. The upper bound so computed was $152 \mathrm{~cm}$. We assume this to be also an upper bound for the maximum distance two robots can transport the heavier $(500 \mathrm{~g})$ prey during the same time period. By comparing the figures, we can see that 


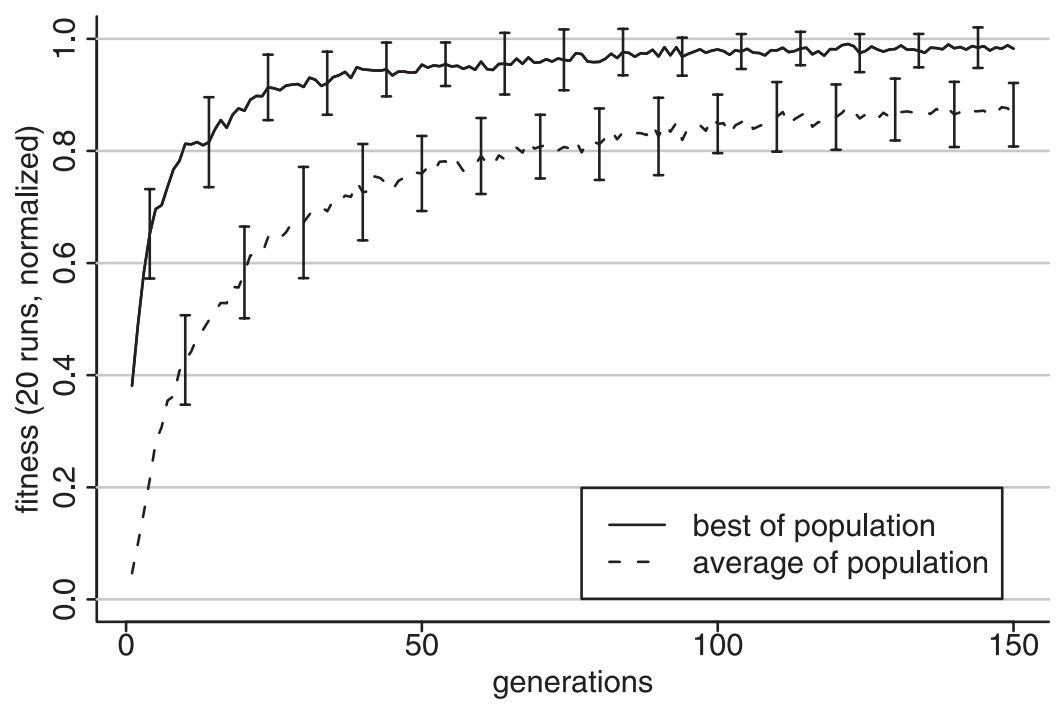

Figure 3 Evolution of transport behaviors with one robot and a 250-g prey. Development of the population best and population average fitness. Each curve corresponds to the average of 20 evolutionary runs with different random seeds. Bars indicate standard deviations.

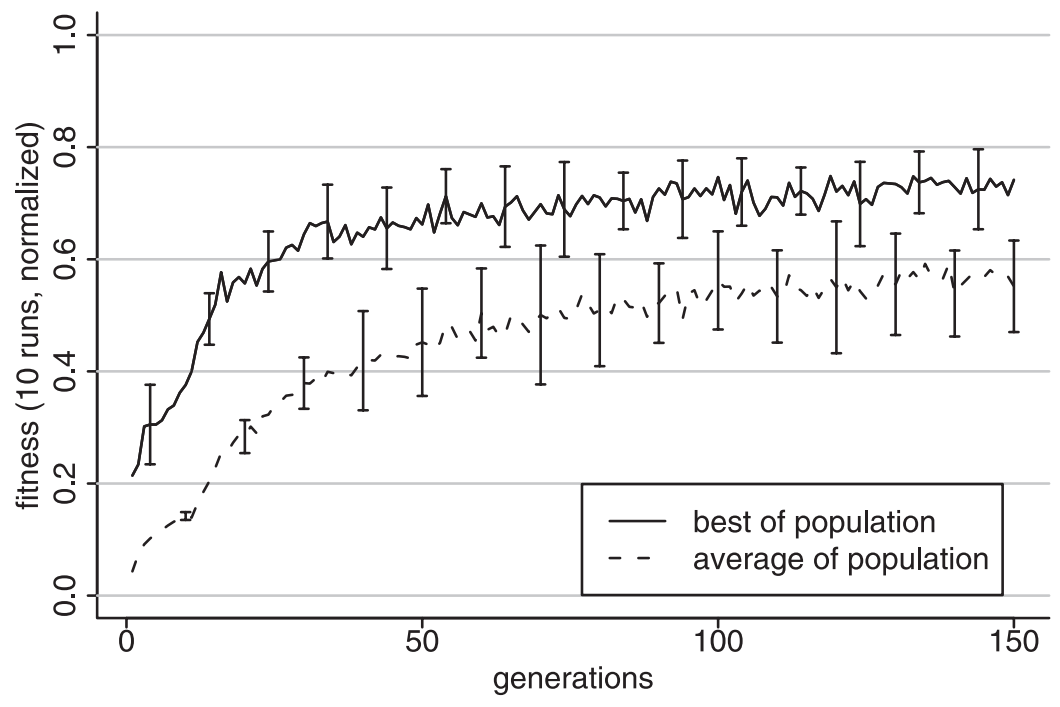

Figure 4 Evolution of transport behaviors with two robots and a 500-g prey. Development of the population best and population average fitness. Each curve corresponds to the average of 10 evolutionary runs with different random seeds. Bars indicate standard deviations.

the fitness values obtained in the one-robot evolutions (see Figure 3) are higher than the fitness values obtained in the two-robot evolutions (see Figure 4).

\subsection{Quantitative Analysis}

The fitness assigned to a group depends not only on the genotype, but also on other factors, including the robots' initial positions and orientations, the position of the light source in the environment, and the noise affecting the robots' sensors and actuators. Thus, there are a very large number of possible configurations to test. However, the genotype is evaluated only in five trials (per generation) during the evolutionary design phase.

To select the best individual of each evolutionary run, we post-evaluate the $\mu=20$ best rated (parent) 


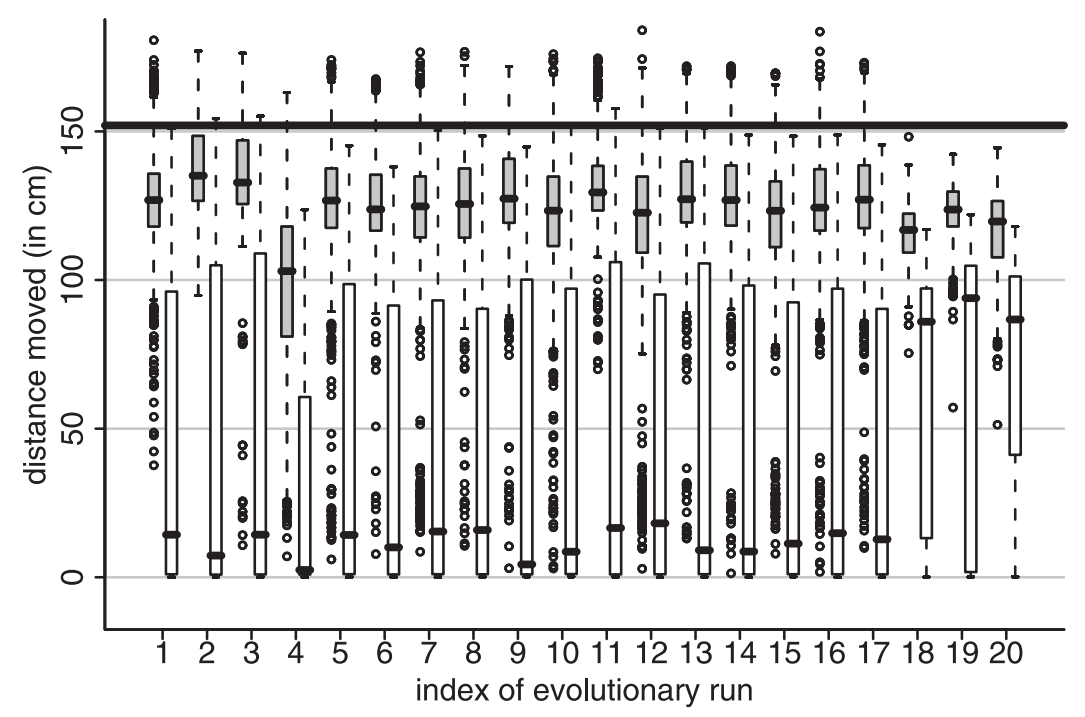

Figure 5 Solitary and group transport performance of the best individuals evolved for solitary transport. Box-andwhisker plot (Becker, Chambers, \& Wilks, 1988) of the distance (in $\mathrm{cm}$ ) the prey was moved by each individual (500 observations per box). Each box comprises observations ranging from the first to the third quartile. The median is indicated by a bar, dividing the box into the upper and lower parts. The whiskers extend to the farthest data points that are within 1.5 times the interquartile range. Outliers are indicated as circles. Characteristics about two types of observations for each individual are displayed: (i) gray boxes refer to solitary transport simulations (one robot, $250 \mathrm{~g}$ prey); (ii) white boxes refer to group transport simulations (two robots, $500 \mathrm{~g}$ prey).

individuals of the final generation on a random sample of 500 start configurations. For every evolutionary run, the individual exhibiting the highest average performance during the post-evaluation is considered to be the best one.

To allow for an unbiased assessment of the performance of the selected individuals, we post-evaluate each of them for a second time on a new random sample of 500 start configurations.

Let us first consider the performance of the best individuals from the evolutionary runs in which a single robot was simulated.

\subsubsection{Individuals Evolved for Solitary Task Per-} formance Figure 5 illustrates the transport performance of the individuals evolved for solitary task performance using a box-and-whisker plot. The gray boxes correspond to the distances (in $\mathrm{cm}$ ) the $250 \mathrm{-g}$ prey was moved by a single robot in the 500 trials of the post-evaluation. The average distances (in $\mathrm{cm}$ ) range from 95.0 to 137.9 . This is $62.5-90.7 \%$ of the upper bound. The standard deviations are in the range $[9.7,35.3]$. Note that the performance in some trials exceeds the upper bound (indicated by the bold hori- zontal line). This is caused by the random differences among the actuators of the robots (e.g., differences in the maximum speed $M$ of a wheel). Recall that to compute the upper bound, any form of random noise was disabled.

We now examine the ability of a group of robotseach acting as in solitary transport-to transport a prey that requires cooperation to be moved. Note that the robots cannot perceive each other, nor have they been trained in situations that involve multiple robots. For each individual, we assessed the performance of a group of two robots on 500 start configurations with the 500-g prey. All robots of the group were initially assigned a copy of the same neural network controller. The results are shown in Figure 5 (white boxes). The average distances (in $\mathrm{cm}$ ) range from 30.4 to 70.1 . This is $20.0-46.1 \%$ of the upper bound. The standard deviations are in the range [38.3, 53.9]. The performance obtained with two robots and the heavy prey is significantly worse than the performance obtained with one robot and the light prey (two-sided MannWhitney test, $5 \%$ significance level).

Let us now consider the performance of the best individuals from the evolutionary runs in which two robots were simulated. 


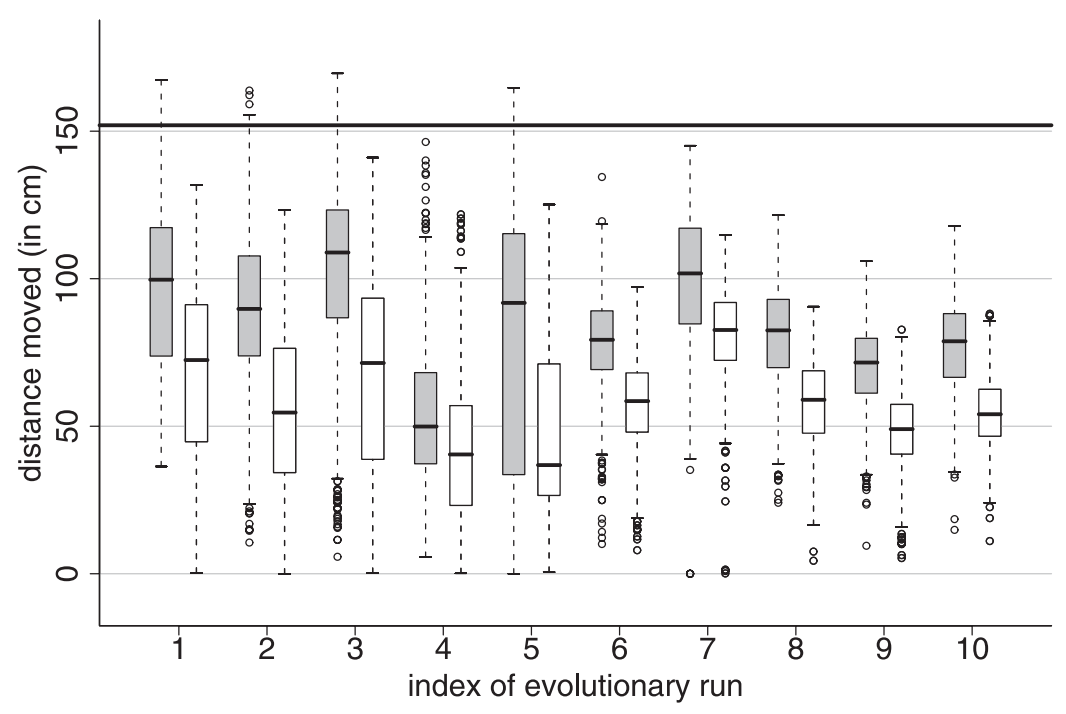

Figure 6 Solitary and group transport performance of the best individuals evolved for group transport. The box-andwhisker plot is explained in the caption of Figure 5.

\subsubsection{Individuals Evolved for Cooperative Task} Performance Figure 6 illustrates the transport performance of the individuals evolved for group transport using a box-and-whisker plot. Once again, we evaluated both the performance in solitary transport and the performance in group transport in 500 trials each. The gray boxes correspond to the distances (in $\mathrm{cm}$ ) the $250-\mathrm{g}$ prey was moved by a single robot. The average distances (in $\mathrm{cm}$ ) range from 53.9 to 101.4. This is $35.4-66.7 \%$ of the upper bound. The standard deviations are in the range [15.1, 40.9]. For the trials with two robots and a 500-g prey (see white boxes), the average distances (in $\mathrm{cm}$ ) range from 41.6 to 80.9. This is $27.4-53.2 \%$ of the upper bound. The standard deviations are in the range $[12.2,35.6]$.

Although during evolution two robots were present, the individuals perform consistently better when tested alone (two-sided Mann-Whitney tests, 5\% significance level). This latter result supports our intuition that group transport is more complex than solitary transport. The presence of multiple robots is likely to lead to interferences that cause a decrease in performance. Moreover, group transport requires a coordinated action as the members of the group have to push or pull the object in similar directions.

With regard to solitary transport performance, the evolutionary algorithm in which solitary transport behavior is selected for generates better performing individuals than the evolutionary algorithm in which group transport behavior is selected for (one-sided Mann-Whitney test, 5\% significance level). With regard to group transport performance, the evolutionary algorithm in which group transport behavior is selected for generates better performing individuals than the evolutionary algorithm in which solitary transport behavior is selected for (one-sided Mann-Whitney test, 5\% significance level).

\subsection{Behavioral Analysis}

In the following, we analyze the behaviors of robots when controlled by the neural networks whose parameters are specified by the individuals evolved for solitary and group transport, respectively. We identify proximate mechanisms that cause the coordination of robots in the group. In particular, we examine the formation of assemblages.

\subsubsection{Individuals Evolved for Solitary Task Per-} formance Concerning the 20 runs for the evolution of solitary transport, 17 out of 20 of the best neural networks let the robot grasp and push the prey by moving forward. Over the 500 trials with two robots in the environment with the 500-g prey, in 96.4$100.0 \%$ of the cases, depending on the neural network used, a robot was connected either directly or indirectly to the prey at the end of the trial. Rarely, self- 
assemblages (i.e., structures of robots being directly connected to each other) were formed (in $0.0-8.8 \%$ of the trials, respectively). In the majority of cases, the robots failed to effectively push the prey in a common direction. The reason for this poor performance is that the robots' behavior and the collective structures they form (via connections with either the prey or with each other) are not suited for the accomplishment of the transport task.

The remaining three neural networks (indexed 18-20 in Figure 5) let the robots push the prey with their bodies by moving backward. These networks display a high median performance, even in group transport. To achieve coordination, the robots do not take advantage of the light source. ${ }^{5}$ Instead, they interact with each other through the prey. If we assume that each robot pushes towards the center of the prey with the same intensity, the combined force of the two exceeds (in intensity) the force of any of the two, as long as their pushing directions differ by less than $120^{\circ}$. As the two robots are initially randomly distributed around the prey, such degree of coordination is present in about two-thirds of the trials. In most of these cases, the resulting force is sufficient to start moving the prey at low speed. As the robots' pushing directions intersect with each other, once the prey is in motion the robots approach each other sliding along the perimeter of the prey. ${ }^{6}$ As the robots continuously adjust their pushing directions according to the position of the prey (and thus to each other), they selforganize into an effective pushing arrangement.

The latter result shows that behaviors that evolve for solitary task performance can provide mutual benefit once robots start acting in groups. In our case, $15 \%$ of the individuals that evolved for solitary transport, once put together with a clone of themselves, exhibit social behavior (mutual benefit); they physically interact with each other (either directly, or through the prey) and thereby enhance their degree of coordination.

\subsubsection{Individuals Evolved for Cooperative Task} Performance Concerning the 10 runs for the evolution of group transport, five out of 10 of the best neural networks let the robots make use of the connection mechanism (corresponding to the first five pairs of boxes in Figure 6). Four out of five neural networks employ the strategy depicted in Figure 7a: each robot cycles (with the connection mechanism heading forward) around the prey to reach a side correlated with the direction of the light source (e.g., the opposite side). Some neural networks let the robot cycle either counterclockwise or clockwise depending on which path is shorter. During this phase, the robot remains distant from the prey, and thereby also from a potential teammate that is already connected to the prey. Once the side that is correlated with the light source is approximately reached, the robot approaches the prey and potentially the connected teammate, and establishes a connection. In other words, by exploiting the relative position of both the prey and the light source, the two robots organize into a dense formation, potentially a linear chain. Each robot keeps on moving forward, pushing the prey (e.g., towards the light source).

The other five neural networks (corresponding to the five latter pairs of boxes in Figure 6) make no use of the connection mechanism. Their strategy is depicted in Figure 7b. They control the robot to move backward. The robot cycles around the prey to reach a side correlated with the light source. Once again, some neural networks let the robot cycle either counterclockwise or clockwise depending on which path is shorter. Differently from the previous behavior, however, the robot comes into physical contact with the prey while cycling around it. In fact, the robot tries to push the prey with its body, while at the same time sliding along the prey's perimeter. If multiple robots are present, their behaviors let them organize into a dense, and thus very effective, pushing arrangement (see Figure 7b).

One neural network was capable of letting the robots display a combination of both types of behaviors (see Figure 7c). In $33.0 \%$ of the cases at the end of the trial, one robot was pushing the prey with the body by moving backward, while the other robot was grasping and pushing the prey, or its teammate, by moving forward (recall that both robots were controlled by an identical neural network). The performance the group achieved in this configuration was significantly higher than the performance the group could achieve in any other configuration when controlled by the same neural network (two-sided Mann-Whitney tests, $5 \%$ significance level).

We examine the physical structures that "emerged" in more detail (only for the five neural networks that let the robots make use of the connection mechanism). Over the 500 trials with two robots in the environment 

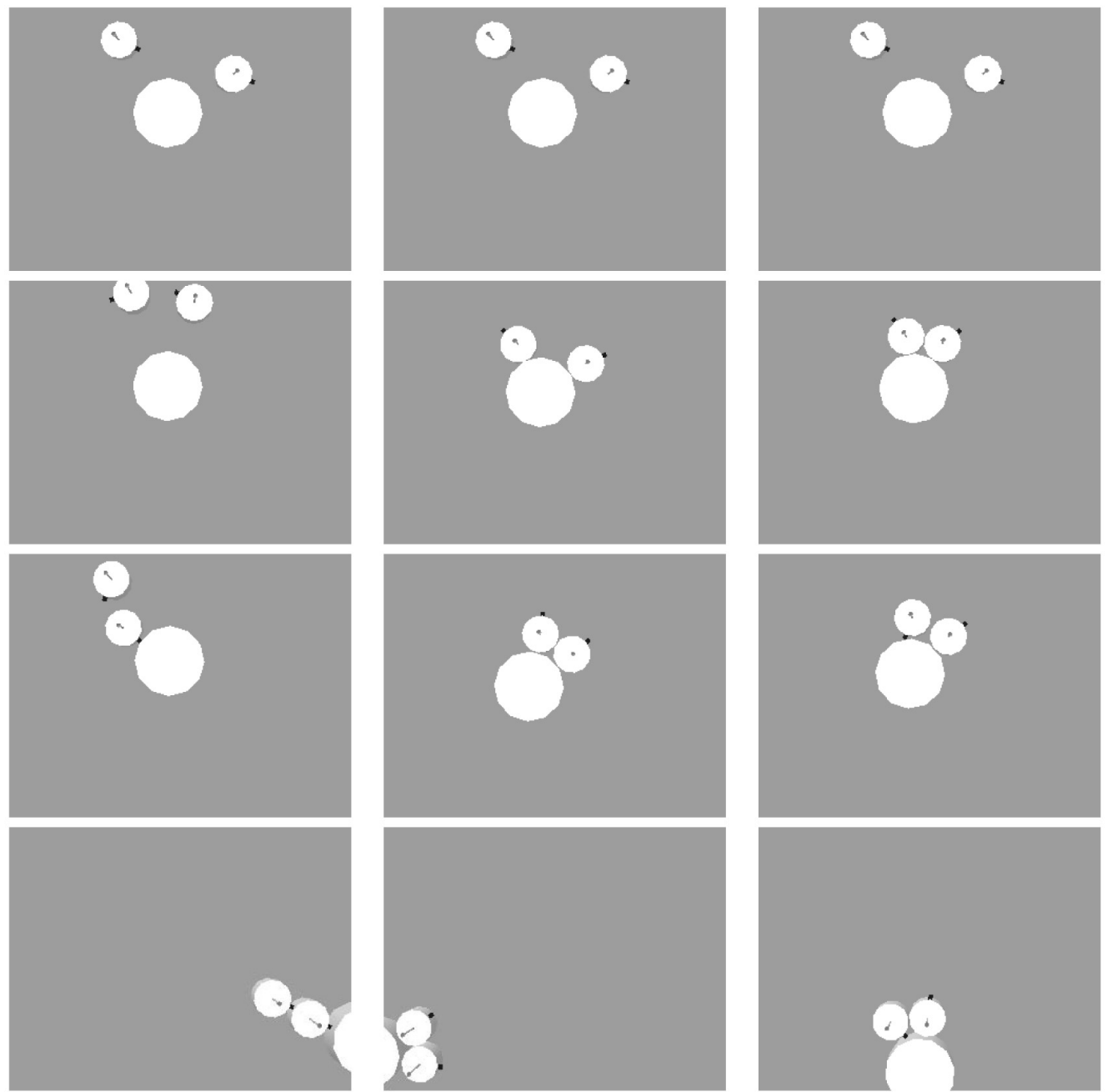

(a)

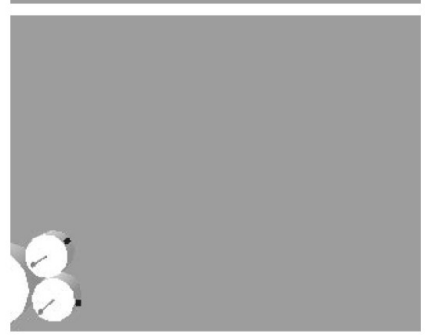

(b)

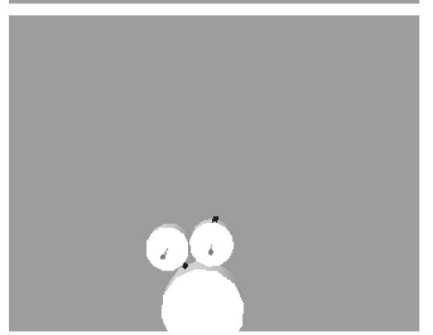

(c)

Figure 7 Group transport of a heavy prey in arbitrary direction. The light source is located outside the range of the image. Both robots are controlled by identical recurrent neural networks. Sequences of actions during a trial (from the top to the bottom, at time $0,2,4$, and $14 \mathrm{~s}$, respectively), corresponding to three different neural networks that respectively (a) let the robots assemble with the prey and/or with the teammate and transport the prey by moving forward, (b) let the robots push the prey with their body by moving backward, (c) let each robot either push the prey by moving backward or assemble with the prey or teammate and push by moving forward.

with the $500-\mathrm{g}$ prey, in $71.1-94.6 \%$ of the cases, depending on the neural network used, a robot was connected either directly or indirectly to the prey at the end of the trial. Self-assembled structures were formed in $6.8-58.8 \%$ of the trials, respectively. Compared to individuals that were evolved for solitary transport (and did also make use of the connection mechanism), the increase in the rate of self-assembly is significant (two-sided Mann-Whitney test, 5\% significance level). For the networks whose strategy is depicted in Figure 7a, self-assembled structures, when formed, were in $77.8-93.5 \%$ of the cases physically attached to the prey. For the network whose strategy is depicted in Figure 7c, the respective value is $42.1 \%$.

\subsection{Scalability}

We examine to what extent the observed behaviors are scalable, that is, whether the evolved individuals are able to let robots cooperate in the transport of a heavier prey when the group size becomes larger. We focus on the best individuals evolved for group trans- 


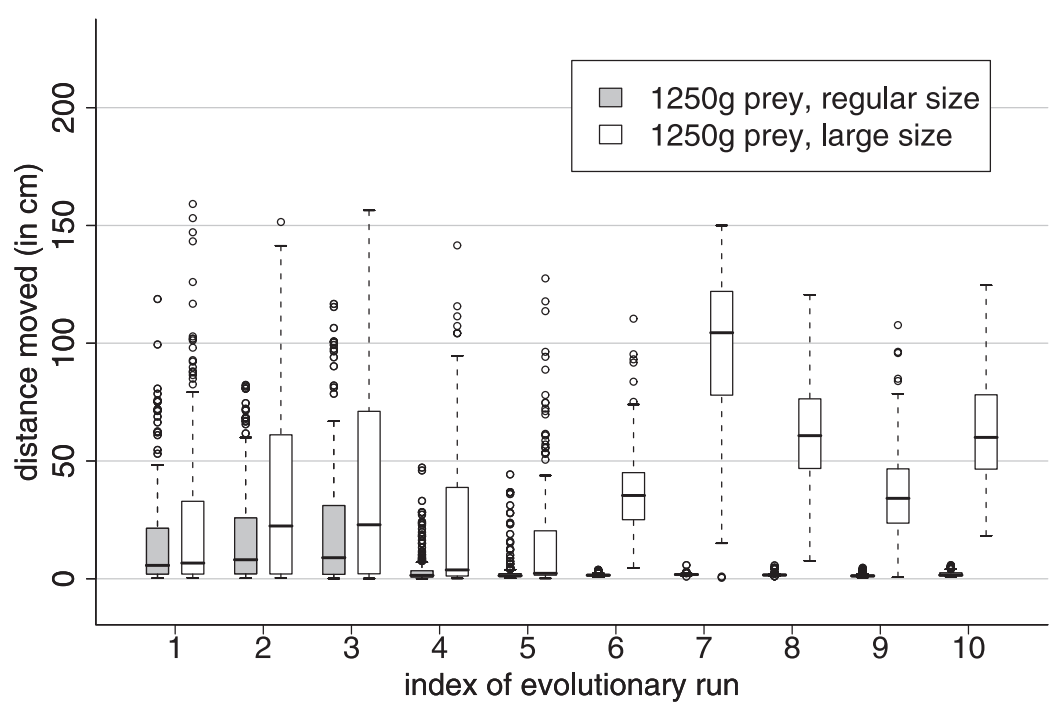

Figure 8 Post-evaluation of the best individuals with groups of five robots transporting a 1250-g prey for $30 \mathrm{~s}$ (200 observations per box). Individuals labeled 1-5 are those that let robots self-assemble; all others make no use of the connection mechanism. Geometry of the prey: (a) size equal to setup during evolution (radius of $12 \mathrm{~cm}$ ), (b) size scaled by the factor the prey's weight as well as the number of robots has increased (radius of $30 \mathrm{~cm}$ ). The box-and-whisker plot is explained in the caption of Figure 5.

port. For each run, we take the best individual and evaluate it 200 times using a group of five robots and a time period of $30 \mathrm{~s}$. We keep the geometry of the prey identical, but we increase its weight proportionally to the increase in the number of robots $(1250 \mathrm{~g})$. The gray boxes of the plot in Figure 8 show the distance (in $\mathrm{cm}$ ) the prey was moved during these trials. The individuals from the first five evolutionary runs shown from the left in the figure are those that let the robots make use of the connection mechanism to solve the task. Self-assemblages occurred in respectively $89.0 \%, 99.0 \%, 92.0 \%, 59.5 \%$, and $46.0 \%$ of the trials. The other five individuals do not let the robots make use of the connection mechanism. Overall, the individuals making use of self-assembly (the average distances are, respectively, 14.4, 18.7, 20.0, 3.7, and 3.4 $\mathrm{cm}$ ) outperform the other individuals (the average distances are, respectively, 1.6, 1.8, 1.7, 1.4, and $2.0 \mathrm{~cm}$ ); two-sided Mann-Whitney test, 5\% significance level. The latter individuals are incapable of achieving the task as the prey does not offer enough contact surface for being pushed effectively by more than two robots (see Figure 9). However, if the perimeter of the prey is scaled by the same factor as the weight and the number of robots has increased, all individuals are able to move the prey, and those that let the robots not self-assemble exhibit a better performance (see white boxes in Figure 8).

Video recordings are available at http://iridia.ulb. ac.be/supp/IridiaSupp2007-007/.

\section{Related Work}

In the following, we briefly review related work on group transport and self-assembly. First, we consider studies that are concerned with groups of social insects and of social spiders. Then, we consider studies that are concerned with groups of robots.

\subsection{Groups of Social Insects and of Social Spiders}

4.1.1 Group Transport In the literature, group transport is almost exclusively reported in the context of ants. In fact, Moffett (1992, p. 220) claimed that group transport "is better developed in ants than in any other animal group." Nevertheless, it "has seldom been recognized as a form of social behavior that is worthy of investigation in its own right" (p. 227).

In most ant species, group transport presumably provides adaptive value as reproductive immatures are 


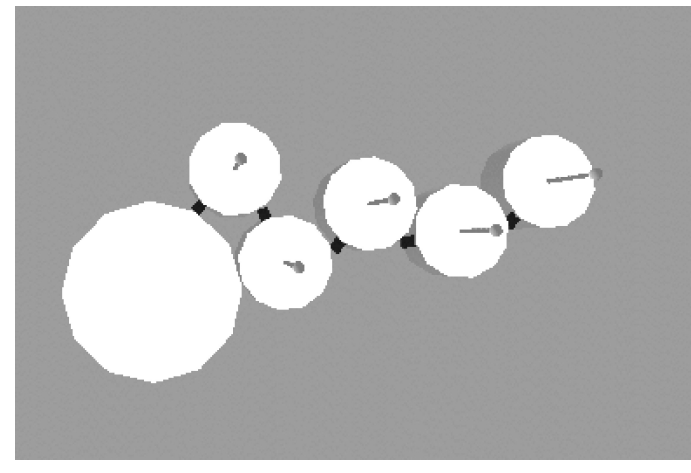

(a)

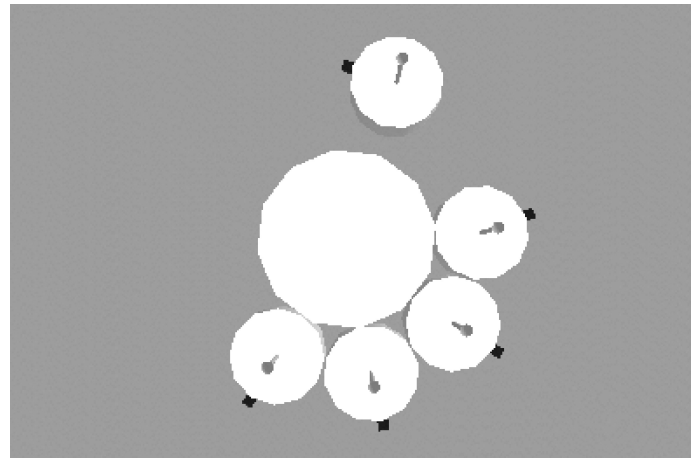

(b)

Figure 9 Group transport of a 1250-g prey (radius of $12 \mathrm{~cm}$ ) by five robots. Snapshots for two different individuals. (a) An individual that let the robots self-assemble. The group is capable of transporting the prey at low speed. (b) An individual that let the robots make no use of the connection mechanism. The group is incapable of moving the prey as the latter offers not enough contact surface for being pushed effectively by more than two robots. For a quantitative analysis see Figure 8.

much bigger than workers, and therefore cannot be transported by a single worker alone (e.g., during an emigration). Moffett (1992, p. 220) states that group transport of "bulky larvae and pupae is probably nearly universal in ants and is likely to have preceded the transport of food by this method". Moffett (1992) lists 39 species of ants for which group transport of food has been reported. He states that "without doubt the group transport of food has arisen independently in numerous phylogenetic lines....At least with regard to carrying food, those ants species capable of group transport are unquestionably in the minority" (p. 227).

Almost half a century ago, Sudd (1960) studied the transport of prey by single ants and by groups of ants of the species Pheidole crassinoda. Sudd reported that during transport the ants did not pull steadily but in short successive hauls that were generally associated with changes in the arrangement of ants in the group. "In almost all series involving groups of ants there was an upward trend of the force exerted in successive hauls; where only one ant was pulling however the proportion of hauls with upward and downward trend was about equal" (p. 301). Changes in the arrangement of ants in the group were of two types (Sudd, 1960):

In realignment the ant altered the orientation of its body without releasing its hold on the prey. Realignment was sometimes the cause and sometimes the effect of rotation of the prey. In repositioning however the ant released the prey and returned to it at a different position. Realignment appeared to correspond to the turning movements of a single ant experiencing difficulty in pulling prey, whilst repositioning corresponded to the excursions which were made from the prey before an ant left it to return to the nest.... Realignment occurred throughout traction but repositioning involved a sharper change and was more occasional (p. 301 and p. 304).

Even though a positive group effect was present, the behavior of individual workers in group transport appeared "to contain no elements of behavior that were not shown by single transporting ants....If cooperative transport existed therefore it resulted from the coordination, within the group, of behavior also shown by individuals working alone" (p. 304).

Franks (1986), Franks, Sendova-Franks, Simmons, and Mogie (1999), and Franks, Sendova-Franks, and Anderson (2001) investigated the performance and organization of groups of army ant workers (Eciton burchellii and Dorylus wilverthi), who cooperate to transport large prey. Army ants carry items by first straddling them so that the item is slung beneath their bodies and, hence, they always face the same direction. In contrast, other ants such as Pheidole crassinoda tend to pull the item, and often several ants pull in different directions. Franks (1986) and Franks et al. (2001) showed that in most of the instances involving the army ants, the group was composed of an unusually large front-runner, which presumably steered and 
determined the direction of transport, and one or more particularly small followers. Anderson and Franks (2001) do not consider the front-runner as a leader in any sense. Instead, they hypothesize that "all of the individuals that form a team in army ants are initially using exactly the same rules of thumb" (p. 537). Franks (1986) reported that the performance in the group was much more than the sum of the performances of its individual members. They could do so probably because by straddling the prey between them the rotational forces (i.e., forces that occur when lifting the prey in a position aside its barycenter) are balanced and disappear.

Superefficiency in group transport has also been observed in other ant species (Hölldobler, Stanton, \& Markl, 1978; Moffett, 1988). In Pheidologeton diversus, for instance, on average an ant engaged in group transport held at least 10 times the weight it did as solitary transporter (Moffett, 1988). Moffett characterized the behaviors in solitary and group transport as follows:

Ants carrying burdens solitarily grasped them between their mandibles, lifting the burdens from the ground and holding the burdens ahead of them as they walked forward (burdens were rarely slung beneath the body). Grouptransporting ants carried burdens differently. One or both forelegs were placed on the burden, and appeared to aid considerably in lifting it. The mandibles were open and usually lay against the burden, but the burden's surface was seldom gripped between them. Burdens small enough to be carried by few ants, as well as small appendages extending from larger burdens, were often carried partly by ants that behaved as described for those engaged in solitary transport (p. 388).

Group transport of prey has also been observed in a few species of social spiders (Vakanas \& Krafft, 2004):

During transport, as an aid to the movement of the prey, spiders weave silk that we named "traction silk", fixed between the prey and the web (in the direction of the shelter) that will permit a slight lifting of the prey. This process will be repeated until the prey has been transported under the shelter (p. 765).

Coordination in group transport by social spiders seems to occur through the item that is transported (Vakanas \& Krafft, 2004):
Movement of one spider engaged in group transport is likely to modify the stimuli perceived by the other group members (such as vibration produced, or indirectly, available site on the prey) possibly producing, in turn, recruitment or departure of individuals. ... Coordination in spider colonies is based on signals that are made inadvertently as side products of their activities. The communal network, as a means of information, seems to be at the origin of cooperation. This supports the hypothesis of a sudden passage from solitary to social life in spinning spiders (Brach, 1977; Wickler \& Seibt, 1993; Plateaux-Quénu, Horel, \& Roland, 1997; Vakanas \& Krafft, 2001) (pp. 770-771).

4.1.2 Self-Assembly Following Whitesides and Grzybowski (2002), self-assembly can be defined as a process by which pre-existing discrete components organize into patterns or structures without human intervention.

Self-assembly has been widely observed in social insects (Anderson et al., 2002; Sendova-Franks \& Franks, 1999). Via self-assembly, ants, bees, and wasps can organize into functional units at an intermediate level between the individual and the colony. Anderson et al. (2002) identified 18 distinct types of self-assembled structures that insects build: "bivouacs, bridges, curtains, droplets, escape droplets, festoons, fills, flanges, ladders, ovens, plugs, pulling chains, queen clusters, rafts, swarms, thermoregulatory clusters, tunnels, and walls" (p. 99). In some cases (e.g., an ant raft) the individuals assemble into "a formless random arrangement," whereas in other cases (e.g., an ant ladder) the individuals assemble into a "particular (required) arrangement" (p. 100). The function of selfassemblages "can be grouped under five broad categories which are not mutually exclusive: (1) defense, (2) pulling structures, (3) thermoregulation, (4) colony survival under inclement conditions, and (5) ease of passage when crossing an obstacle" (p. 99). Anderson et al. (2002) claim that in almost all of the observed instances, the function could not be achieved without self-assembly.

Pulling structures have been observed in a few ant species (e.g., Eciton burchellii) as well as in honey bees (Apis mellifera; Anderson et al., 2002). The structures formed generate torque, for instance, to fix a large prey to the floor or to bend a leaf during nest construction. Although a pulling structure may only 
require a few individuals, often a critical density of individuals may be required to initiate self-assembly and growth (Anderson et al., 2002). When part of an assembled structure, ants have few degrees of mobility. In some species, worker ants seem even to become motionless as a reaction to being stretched (see Anderson et al., 2002 and references therein).

At present "virtually nothing is known regarding the rules, signals, and cues used by individuals in formation [of assembled structures] or the physical constraints these structures are under" (Anderson et al., 2002, p. 107). Lioni and Deneubourg (2004) and Lioni, Sauwens, Theraulaz, and Deneubourg (2001) studied mechanisms by which ants of the genus Ecophylla form living ladders and bridges by linking with each other. They showed that the ants, if offered two alternative sites to bridge an empty space, typically end up in a single, large aggregate in either one of the two sites. They observed that the process is controlled by the individual probabilities of entering and leaving the aggregates. The probabilities depend on the number of ants in the aggregate.

Theraulaz et al. (2001) modeled self-assembly processes in Linepithema humile using an agent-based approach. The ants aggregated at the end of a rod and formed droplets containing several assembled ants that eventually fell down. The model could be tuned to reproduce some properties of the experimental system, such as the droplet size and the inter-drop interval. The function of this behavior is currently unknown.

\subsection{Groups of Robots}

4.2.1 Group Transport In most studies of transport in robotic groups, the members of the group move an object by pushing it. Pushing strategies have the advantage that they allow the robots to move objects that are hard to grasp. In addition, multiple objects can be pushed at the same time. However, it is difficult to predict the motion of the object and of the robots, especially if the ground is not uniform. ${ }^{7}$ Therefore, the control typically requires sensory feedback. Most studies consider two robots pushing a wide box simultaneously from a single side (Donald, Jennings, \& Rus, 1997; Gerkey \& Matarić, 2002; Matarić, Nilsson, \& Simsarian, 1995; Parker, 1999; Sugie, Inagaki, Ono, Aisu, \& Unemi, 1995). To coordinate the robots' actions, robots are specifically arranged (Donald et al., 1997; Gerkey \& Matarić, 2002; Matarić et al., 1995;
Parker, 1999), control is synchronized (Matarić et al., 1995) relative positions are known (Donald et al., 1997; Parker, 1999), explicit communication is used (Matarić et al., 1995; Parker, 1999), and/or individual tasks are generated by a designated leader agent (Gerkey \& Matarić, 2002; Sugie et al., 1995).

Only a few studies have considered more than two robots, pushing a box simultaneously (e.g., Kube \& Bonabeau, 2000; Kube \& Zhang, 1993, 1997; Yamada $\&$ Saito, 2001). In these cases, the control is homogeneous and decentralized; the robots make no use of explicit communication. Kube and Zhang (1997) and Kube and Bonabeau (2000) reported that if the box is small compared to the size of the pushing robots, the performance decreases drastically with group size as the box offers only limited contact surface.

Many studies have considered the transport of an object by multiple, mobile robots grasping and/or lifting it. In these studies, typically two to three robots are manually attached to the object (Aiyama, Hara, Yabuki, Ota, \& Arai, 1999; Desai, Wang, Žefran, \& Kumar, 1996; Kosuge \& Oosumi, 1996; Miyata, Ota, Arai, \& Asama, 2002; Sugar \& Kumar, 2002; Wang, Nakano, \& Takahashi, 2003). To coordinate the robots' actions, often, robots have knowledge of their relative positions. In some systems, the desired trajectories are given prior to experimentation to all robots of the group. The object is transported as each robot follows the given trajectory by making use of dead-reckoning (Desai et al., 1996). In other systems, the manipulation is planned in real time by an external workstation, which communicates with the robots (Miyata et al., 2002). Often, instead of an external computer, a specific robot called the "leader" knows the desired trajectory or the goal location. The leader robot can send explicit high- or low-level commands to the "followers" (Sugar \& Kumar, 2002; Wang et al., 2003). However, in many leader-follower systems explicit communication is not required (Aiyama et al., 1999; Kosuge \& Oosumi, 1996).

4.2.2 Self-Assembly Self-reconfigurable robots (Rus, Butler, Kotay, \& Vona, 2002; Yim, Zhang, \& Duff, 2002) hold the potential to self-assemble and thus to mimic the complex behavior of social insects. In current implementations (Jørgensen, Østergaard, \& Lund, 2004; Murata et al., 2002; Rus et al., 2002; Yim et al., 2002), however, single modules usually have 
highly limited autonomous capabilities (when compared to an insect). Typically, they are not equipped with sensors to perceive the environment. Nor, typically, are they capable of autonomous motion. These limitations, common to most self-reconfigurable robotic systems, make it difficult to let separate modules, or groups of modules, connect autonomously. In some systems, self-assembly was demonstrated with the modules being pre-arranged at known positions (Yim, Zhang, Roufas, Duff, \& Eldershaw, 2002; Zykov, Mytilinaios, Adams, \& Lipson, 2005). Some instances of less constrained self-assembly have been reported (for an overview, see Groß \& Dorigo, in press). Fukuda, Nakagawa, Kawauchi, and Buss (1988) and Fukuda, Ueyama, and Sekiyama (1995) demonstrated self-assembly among robotic cells using the CEBOT system (Fukuda \& Ueyama, 1994). In the experiment, a moving cell approached and connected with a static cell. The moving cell was controlled with a finite-state automata. Rubenstein, Payne, Will, and Shen (2004) demonstrated the ability of two modular robots to selfassemble. Each robot consisted of a chain of two linearly-linked CONRO modules (Castano, Behar, \& Will, 2002). The robot chains were set up at distances of $15 \mathrm{~cm}$, facing each other with an angular displacement not larger than $45^{\circ}$. The control was heterogeneous, both at the level of individual modules within each robot and at the level of the modular makeup of both robots. Trianni, Tuci, and Dorigo (2004) evolved a neural network controller for individual and collective phototaxis. The results obtained using embodied simulations demonstrate that groups of up to three mobile robots while performing phototaxis can selfassemble and disassemble in response to the temperature of the environment. The use of self-assembly was explicitly rewarded by the fitness function. None of these studies demonstrates the utility of self-assembling robots for a concrete task.

\section{Discussion and Conclusions}

In this article we have studied the evolution of cooperation by investigating the relation between solitary and social behavior; the question that is addressed is to what extent individuals that as a group have to accomplish a cooperative task can benefit from behaving differently from individuals that have to accomplish tasks on their own. Related work from biology suggests that, in some species, individuals show no difference in behavior when engaged in solitary and group transport. Following this, we hypothesize that group transport in some species evolved from solitary transport.

We designed a study that allows for some initial test on the plausibility of such a transition from solitary to social behavior. In particular, we studied the solitary and group transport of an object called prey using physics-based computer simulations. We simulated simplistic robots that, similarly to ants, can move autonomously and grasp objects in their environment. The robots' cognitive capabilities were very limited and similar to those of many solitary animals: they were not capable of perceiving potential teammates or of communicating with them. We performed two sets of experiments to produce (using evolutionary algorithms) neural networks that control robots when competing based on solitary task performance and cooperative task performance.

Each of the evolutionary runs we performed (30 in total) yielded an individual of satisfactory performance (the individuals achieved $62.5-90.7 \%$ and $27.4-53.2 \%$ of an upper bound performance for solitary transport and group transport, respectively). Networks evolved for solitary task performance were capable of letting robots engage also in group transport (the individuals achieved $20.0-46.1 \%$ of the upper bound performance). Networks evolved for group transport were capable of letting robots engage also in solitary transport (the individuals achieved $35.4-66.7 \%$ of the upper bound performance). Our results show also that group transport and solitary transport impose different demands on the robot. In fact, robots engaged in group transport benefit from behaving differently from those engaged in solitary transport. The results support also our intuition that group transport is a more complex task than solitary transport. Furthermore, our study revealed a variety of proximate mechanisms that can cause coordinated behavior. In particular, we observed that robots enhance their degree of coordination by physically interacting either directly or indirectly (i.e., via the environment) and by exploiting the presence of visual cues in the environment. This holds not only for all networks evolved for cooperative task performance, but also for $15 \%$ of the networks evolved for solitary task performance. That is, some "solitary individuals," when grouped together with a clone, exhibit social behavior (mutual benefit). 
In half of the evolutionary runs with groups of robots, the best-rated neural networks let robots selfassemble. Self-assembled structures among the robots were formed in up to $58.8 \%$ of the trials with two robots and in up to $99.0 \%$ of the trials with five robots. Note that the fitness function of the evolutionary algorithm neither explicitly rewarded the robots for selfassembling, nor did it impose any bias concerning the spatial organization of the robots during task performance. The "emergence" of self-assembly is a striking result, confirming that such capability (as in social insects) can provide adaptive value to the group. The analysis revealed the proximate mechanisms that caused the formation of the self-assemblages. In particular, two visual cues present in the environment (the prey and a light source) were sufficient to guide robots in the formation of assembled structures. Moreover, as the assemblages were formed by robots facing in approximately the same direction (and sometimes opposite directions), they were suitable for the accomplishment of the task (which required all robots to pull/push the prey in approximately the same direction). When increasing both the group size and the weight of the object, the performance decreased. Groups that selfassembled were still capable of moving the prey. Groups that did not make use of self-assembly were incapable of moving the prey, unless the prey size was increased proportionally.

Overall, our work supports the hypothesis that in some species group transport has evolved from solitary transport, presumably from situations in which solitary transporters, without being aware of each other, cooperatively transported a common load. Because such behaviors could provide adaptive value, it is likely they became improved through evolution (which potentially led to specialized behaviors for group transport). To the best of our knowledge, although plausible, this hypothesis has not been further investigated by biologists. Such transition would require (as was the case in our study) that multiple individuals were likely to interact with a same load, and that these individuals were related to each other. Future work will investigate more comprehensive models (e.g., including the spatial distribution of competing colonies, and the spatial distribution of different types of prey), and compare the results against knowledge on biological systems.

From an engineering perspective, our work showed that cooperative behavior can be achieved by simple means-the robots could neither perceive nor commu- nicate with each other directly. Nevertheless, they could organize into effective transport arrangements.

In general, the study of artificial systems, in which self-assembly evolves as providing some functional benefit to the interacting individuals, can offer invaluable insight into biological self-assembling systems. It is also a promising direction to be further explored in the evolutionary design of robotic systems. Our work has provided some first evidence that self-assembly can provide adaptive value to a group of robots. The value certainly depends on many factors (e.g., mass and size of the prey, number and size of the robots). In ants, self-assembled structures are rarely observed in group transport (yet they are relevant in other contexts). Individual limitations might prevent individuals, which are organized into an assemblage, from moving collectively by their own propulsion (see Section 4). This could explain why self-assemblages are virtually non-existent for group transport in ants. Our scalability analysis indicates that our simple robots might be subject to similar limitations when the group size is increased. For designing group transport systems, more capable robotic hardware can be explored. Following this approach, we have designed group transport systems that are effective also for larger group sizes (Groß \& Dorigo, 2004a, 2004b; Groß, Mondada, \& Dorigo, 2006; Groß, Tuci, et al., 2006; Tuci et al., 2006).

\section{Acknowledgments}

This work was supported by the IST FET project "SWARMBOTS" (in the form of grant number IST-2000-31010), and by the "ANTS" project, an "Action de Recherche Concertée" funded by the Scientific Research Directorate of the French Community of Belgium. RG acknowledges support from the BBSRC (in the form of grant number E19832). MD acknowledges support from the Belgian F.R.S.-FNRS, of which he is a research director. The authors thank three anonymous referees, Christos Ampatzis, and Nigel R. Franks for helpful comments on a previous version of this article.

\section{Notes}

1 Randomness affects properties of the robot (e.g., its perceptual range). This may account for imprecision associated with building robotic hardware, and is not genetically determined.

2 On the physical s-bot robot, instead, we obtain information on angles and distances by processing the image taken from the camera by feature extraction algorithms 
(Groß, Bonani, Mondada, \& Dorigo, 2006; Nouyan, Groß, Bonani, Mondada, \& Dorigo, 2006).

3 For simplicity, by individual we refer to the genotype. Note that the genotype encodes a neural network controller, which is cloned and copied to each robot of a group.

4 Note that the computational costs may increase superlinearly with the number of robots being simulated. This is particularly the case if the robots physically interact with each other.

5 Only the neural network from run 20 lets the robots (slightly) correlate their direction of pushing with the direction of the light source. The networks from runs 18 and 19, however, do not let the robots correlate their direction of pushing with the direction of the light source. In fact, they let the robots transport the prey in a direction that is uniformly random (as experimentally verified). Recall that the task is to move the prey, the farther the better, in an arbitrary direction.

6 Recall that the particular behavior that is discussed here lets the robots make no use of their connection mechanisms. Instead, the robots' bodies are in physical contact with the prey and push the latter by moving backward.

7 For a theory on the mechanics of pushing, see Mason (1986).

\section{References}

Aiyama, Y., Hara, M., Yabuki, T., Ota, J., \& Arai, T. (1999). Cooperative transportation by two four-legged robots with implicit communication. Robotics and Autonomous Systems, 29(1), 13-19.

Anderson, C., \& Franks, N. R. (2001). Teams in animal societies. Behavioral Ecology, 12(5), 534-540.

Anderson, C., \& McShea, D. W. (2001). Intermediate-level parts in insect societies: Adaptive structures that ants build away from the nest. Insectes Sociaux, 48(4), 291-301.

Anderson, C., Theraulaz, G., \& Deneubourg, J.-L. (2002). Selfassemblages in insect societies. Insectes Sociaux, 49(2), 99-110.

Baldassarre, G., Nolfi, S., \& Parisi, D. (2003). Evolving mobile robots able to display collective behaviour. Artificial Life, 9(3), 255-267.

Becker, R. A., Chambers, J. M., \& Wilks, A. R. (1988). The new $S$ language. A programming environment for data analysis and graphics. London: Chapman and Hall.

Beyer, H.-G. (2001). The theory of evolution strategies. Berlin, Springer.

Bourke, A. F. G., \& Franks, N. R. (1995). Social evolution in ants. Princeton, NJ: Princeton University Press.

Brach, V. (1977). Anelosimus studiosus (Araneae: Theridiidae) and the evolution of quasisociality in theridiid spiders. Evolution, 31(1), 154-161.

Camazine, S., Deneubourg, J.-L., Franks, N. R., Sneyd, J., Theraulaz, G., \& Bonabeau, E. (2001). Self-organization in biological systems. Princeton, NJ: Princeton University Press.
Castano, A., Behar, A., \& Will, P. M. (2002). The Conro modules for reconfigurable robots. IEEE/ASME Transactions on Mechatronics, 7(4), 403-409.

Coulomb, C. A. (2001). Théorie des machines simples, en ayant égard au frottement de leurs parties et à la roideur des cordages [Theory of simple machines with regard for the friction of their parts and the tension of the ropes] (in French). Boston: Adamant Media Corporation. (Reprint of an 1821 edition by Bachelier, Paris.)

Dawkins, R. (1976). The selfish gene. New York: Oxford University Press.

Desai, J., Wang, C.-C., Žefran, M., \& Kumar, V. (1996). Motion planning for multiple mobile manipulators. In Proceedings of the 1996 IEEE International Conference on Robotics and Automation (Vol. 3, pp. 2073-2078). Los Alamitos, CA: IEEE Computer Society Press.

Donald, B. R., Jennings, J., \& Rus, D. (1997). Information invariants for distributed manipulation. International Journal of Robotics Research, 16(5), 673-702.

Dorigo, M. (2005). Swarm-bot: An experiment in swarm robotics. In P. Arabshahi \& A. Martinoli (Eds.), Proceedings of the 2005 IEEE Swarm Intelligence Symposium (pp. 192200). Los Alamitos, CA: IEEE Computer Society Press.

Dorigo, M., Trianni, V., Şahin, E., Groß, R., Labella, T. H., Baldassarre, G., et al. (2004). Evolving self-organizing behaviors for a swarm-bot. Autonomous Robots, 17(2-3), 223-245.

Dorigo, M., Tuci, E., Trianni, V., Groß, R., Nouyan, S., Ampatzis, C., et al. (2006). SWARM-BOT: Design and implementation of colonies of self-assembling robots. In G. Y. Yen \& D. B. Fogel (Eds.), Computational intelligence: Principles and practice (pp. 103-135). Los Alamitos, CA: IEEE Computer Society Press.

Elman, J. L. (1990). Finding structure in time. Cognitive Science, 14(2), 179-211.

Floreano, D., Mitri, S., Magnenat, S., \& Keller, L. (2007). Evolutionary conditions for the emergence of communication in robots. Current Biology, 17(6), 514-519.

Foster, K. R., Wenseleers, T., \& Ratnieks, F. L. W. (2006). Kin selection is the key to altruism. Trends in Ecology and Evolution, 21(2), 57-60.

Franks, N. R. (1986). Teams in social insects: Group retrieval of prey by army ants (Eciton burchelli, Hymenoptera: Formicidae). Behavioral Ecology and Sociobiology, 18(6), 425-429.

Franks, N. R., Sendova-Franks, A. B., \& Anderson, C. (2001). Division of labour within teams of new world and old world army ants. Animal Behaviour, 62(4), 635-642.

Franks, N. R., Sendova-Franks, A. B., Simmons, J., \& Mogie, M. (1999). Convergent evolution, superefficient teams and tempo in Old and New World army ants. Proceedings of the Royal Society B - Biological Sciences, 266(1429), 1697-1701. 
Fukuda, T., Nakagawa, S., Kawauchi, Y., \& Buss, M. (1988). Self-organizing robots based on cell structures-CEBOT. In Proceedings of the 1988 IEEE International Workshop on Intelligent Robots (pp. 145-150). Los Alamitos, CA: IEEE Computer Society Press.

Fukuda, T., \& Ueyama, T. (1994). Cellular robotics and micro robotic systems. Singapore: World Scientific.

Fukuda, T., Ueyama, T., \& Sekiyama, K. (1995). Distributed intelligent systems in cellular robotics. In S. G. Tzafestas \& H. B. Verbruggen (Eds.), Artificial intelligence in industrial decision making, control and automation (pp. 225-246). Dordrecht: Kluwer Academic.

Garnier, S., Gautrais, J., \& Theraulaz, G. (2007). The biological principles of swarm intelligence. Swarm Intelligence, 1(1), 3-31.

Gerkey, B. P., \& Matarić, M. J. (2002). Sold!: Auction methods for multirobot coordination. IEEE Transactions on Robotics and Automation, 18(5), 758-768.

Grassé, P.-P. (1959). La reconstruction du nid et les coordinations interindividuelles chez Bellicositermes natalensis et Cubitermes sp. La théorie de la stigmergie: Essai d'interprétation du comportement des termites constructeurs [Nest rebuilding and coordination between individuals for Bellicositermes natalensis and Cubitermes sp. The theory of stigmergy: An attempt to interpret the behavior of builder termites] (in French). Insectes Sociaux, 6(1), 41-80.

Groß, R., Bonani, M., Mondada, F., \& Dorigo, M. (2006). Autonomous self-assembly in swarm-bots. IEEE Transactions on Robotics, 22(6), 1115-1130.

Groß, R., \& Dorigo, M. (2004a). Cooperative transport of objects of different shapes and sizes. In M. Dorigo et al. (Eds.), Proceedings of the 4th International Workshop on Ant Colony Optimization and Swarm Intelligence, Lecture notes in computer sciences (Vol. 3172, pp. 107-118). Berlin: Springer.

Groß, R., \& Dorigo, M. (2004b). Group transport of an object to a target that only some group members may sense. In X. Yao et al. (Eds.), Proceedings of the 8th International Conference on Parallel Problem Solving from Nature, Lecture notes in computer sciences (Vol. 3242, pp. 852861). Berlin: Springer.

Groß, R., \& Dorigo, M. (in press). Self-assembly at the macroscopic scale. Proceedings of the IEEE.

Groß, R., Mondada, F., \& Dorigo, M. (2006). Transport of an object by six pre-attached robots interacting via physical links. In Proceedings of the 2006 IEEE International Conference on Robotics and Automation (pp. 1317-1323). Los Alamitos, CA: IEEE Computer Society Press.

Groß, R., Tuci, E., Dorigo, M., Bonani, M., \& Mondada, F. (2006). Object transport by modular robots that selfassemble. In Proceedings of the 2006 IEEE International Conference on Robotics and Automation (pp. 2558-2564). Los Alamitos, CA: IEEE Computer Society Press.
Hamilton, W. D. (1964). The genetic evolution of social behaviour. I. Journal of Theoretical Biology, 7(1), 1-16.

Harvey, I., Husbands, P., Cliff, D., Thompson, A., \& Jakobi, N. (1997). Evolutionary robotics: The Sussex approach. Robotics and Autonomous Systems, 20(2-4), 205-224.

Hölldobler, B., Stanton, R. C., \& Markl, H. (1978). Recruitment and food-retrieving behavior in Novomessor (Formicidae, Hymenoptera). Behavioral Ecology and Sociobiology, 4(2), 163-181.

Jørgensen, M. W., Østergaard, E. H., \& Lund, H. H. (2004). Modular ATRON: Modules for a self-reconfigurable robot. In Proceedings of the 2004 IEEE/RSJ International Conference on Intelligent Robots and Systems (Vol. 2, pp. 20682073). Los Alamitos, CA: IEEE Computer Society Press.

Klumpp, S., \& Lipowsky, R. (2005). Cooperative cargo transport by several molecular motors. Proceedings of the National Academy of Sciences of the United States of America, 102(48), 17284-17289.

Korb, J., \& Heinze, J. (2004). Multilevel selection and social evolution of insect societies. Naturwissenschaften, 91(6), 291-304.

Kosuge, K., \& Oosumi, T. (1996). Decentralized control of multiple robots handling an object. In Proceedings of the 1996 IEEE/RSJ International Conference on Intelligent Robots and Systems (Vol. 1, pp. 318-323). Los Alamitos, CA: IEEE Computer Society Press.

Kube, C. R., \& Bonabeau, E. (2000). Cooperative transport by ants and robots. Robotics and Autonomous Systems, 30(1-2), 85-101.

Kube, C. R., \& Zhang, H. (1993). Collective robotics: From social insects to robots. Adaptive Behavior, 2(2), 189-218.

Kube, C. R., \& Zhang, H. (1997). Task modelling in collective robotics. Autonomous Robots, 4(1), 53-72.

Liang, K.-H., Yao, X., Liu, Y., Newton, C. S., \& Hoffman, D. (1998). An experimental investigation of self-adaptation in evolutionary programming. In V. W. Porto, N. Saravanan, D. Waagen, \& A. E. Eiben (Eds.), Proceedings of the 7th International Conference on Evolutionary Programming, Lecture notes in computer sciences (Vol. 1447, pp. 291-300). Berlin: Springer.

Lioni, A., \& Deneubourg, J.-L. (2004). Collective decision through self-assembling. Naturwissenschaften, 91(5), 237-241.

Lioni, A., Sauwens, C., Theraulaz, G., \& Deneubourg, J.-L. (2001). Chain formation in Ecophylla longinoda. Journal of Insect Behavior, 14(5), 679-696.

Martinoli, A. (1999). Swarm intelligence in autonomous collective robotics: From tools to the analysis and synthesis of distributed control strategies. Ph.D. thesis, EPFL, Lausanne, Switzerland.

Mason, M. T. (1986). Mechanics and planning of manipulator pushing operations. International Journal of Robotics Research, 5(3), 53-71. 
Matarić, M. J., Nilsson, M., \& Simsarian, K. T. (1995). Cooperative multi-robot box-pushing. In Proceedings of the 1995 IEEE/RSJ International Conference on Intelligent Robots and Systems (Vol. 3, pp. 556-561). Los Alamitos, CA: IEEE Computer Society Press.

Miyata, N., Ota, J., Arai, T., \& Asama, H. (2002). Cooperative transport by multiple mobile robots in unknown static environments associated with real-time task assignment. IEEE Transactions on Robotics and Automation, 18(5), 769-780.

Moffett, M. W. (1988). Cooperative food transport by an Asiatic ant. National Geographic Research, 4(3), 386-394.

Moffett, M. W. (1992). Ant foraging. National Geographic Research and Exploration, 8(2), 220-231.

Mondada, F., Gambardella, L. M., Floreano, D., Nolfi, S., Deneubourg, J.-L., \& Dorigo, M. (2005). The cooperation of swarm-bots: Physical interactions in collective robotics. IEEE Robotics and Automation Magazine, 12(2), 21-28.

Murata, S., Yoshida, E., Kamimura, A., Kurokawa, H., Tomita, K., \& Kokaji, S. (2002). M-TRAN: Self-reconfigurable modular robotic system. IEEE/ASME Transactions on Mechatronics, 7(4), 431-441.

Nolfi, S., \& Floreano, D. (2000). Evolutionary robotics-the biology, intelligence, and technology of self-organizing machines. Cambridge, MA: MIT Press.

Nouyan, S., Groß, R., Bonani, M., Mondada, F., \& Dorigo, M. (2006). Group transport along a robot chain in a selforganised robot colony. In T. Arai, R. Pfeifer, T. Balch, \& H. Yokoi (Eds.), Proceedings of the 9th International Conference on Intelligent Autonomous Systems (pp. 433442). Amsterdam: IOS Press.

Panait, L., \& Luke, S. (2003). Learning ant foraging behaviors. In J. Pollack, M. Bedau, P. Husbands, T. Ikegami, \& R. A. Watson (Eds.), Proceedings of the 9th International Conference on the Simulation and Synthesis of Living Systems (pp. 569-574). Cambridge, MA: MIT Press.

Parker, L. E. (1999). Adaptive heterogeneous multi-robot teams. Neurocomputing, 28(1-3), 75-92.

Pérez-Uribe, A., Floreano, D., \& Keller, L. (2003). Effects of group composition and level of selection in the evolution of cooperation in artificial ants. In W. Banzhaf, T. Christaller, P. Dittrich, J. T. Kim, \& J. Ziegler (Eds.), Proceedings of the 7th European Conference on Artificial Life, Lecture notes in artificial intelligence (Vol. 2801, pp. 128-137). Berlin: Springer.

Plateaux-Quénu, C., Horel, A., \& Roland, C. (1997). A reflection on social evolution in two different groups of arthropods: halictine bees (Hymenoptera) and spiders (Arachnida). Ethology, Ecology and Evolution, 9(2), 183-196.

Pugh, J., \& Martinoli, A. (2006). Multi-robot learning with particle swarm optimization. In P. Stone \& G. Weiss (Eds.), Proceedings of the 5th International Joint Conference on Autonomous Agents and Multiagent Systems (pp. 441448). New York: ACM Press.
Quinn, M., Smith, L., Mayley, G., \& Husbands, P. (2003). Evolving controllers for a homogeneous system of physical robots: Structured cooperation with minimal sensors. Philosophical Transactions of the Royal Society A Mathematical Physical and Engineering Sciences, 361(1811), 2321-2343.

Reynolds, C. W. (1993). An evolved, vision-based behavioral model of coordinated group motion. In J.-A. Meyer, H. L. Roitblat, \& S. W. Wilson (Eds.), Proceedings of the 2 nd International Conference on Simulation of Adaptive Behavior (pp. 384-392). Cambridge, MA: MIT Press.

Rubenstein, M., Payne, K., Will, P., \& Shen, W.-M. (2004). Docking among independent and autonomous CONRO self-reconfigurable robots. In Proceedings of the 2004 IEEE International Conference on Robotics and Automation (Vol. 3, pp. 2877-2882). Los Alamitos, CA: IEEE Computer Society Press.

Rus, D., Butler, Z., Kotay, K., \& Vona, M. (2002). Self-reconfiguring robots. Communications of the ACM, 45(3), 39-45.

Schwefel, H.-P. (1975). Evolutionsstrategie und numerische Optimierung [Evolution strategy and numerical optimization] (in German). Ph.D. thesis, Fachbereich Verfahrenstechnik, Technische Universität Berlin, Berlin, Germany.

Sendova-Franks, A. B., \& Franks, N. R. (1999). Self-assembly, self-organization and division of labour. Philosophical Transactions of the Royal Society B - Biological Sciences, 354(1388), 1395-1405.

Spector, L., Klein, J., Perry, C., \& Feinstein, M. (2005). Emergence of collective behavior in evolving populations of flying agents. Genetic Programming and Evolvable Machines, 6(1), 111-125.

Sudd, J. H. (1960). The transport of prey by an ant, Pheidole crassinoda Em. Behaviour, 16, 295-308.

Sugar, T. G., \& Kumar, V. (2002). Control of cooperating mobile manipulators. IEEE Transactions on Robotics and Automation, 18(1), 94-103.

Sugie, H., Inagaki, Y., Ono, S., Aisu, H., \& Unemi, T. (1995). Placing objects with multiple mobile robots-mutual help using intention inference. In Proceedings of the 1995 IEEE International Conference on Robotics and Automation (Vol. 2, pp. 2181-2186). Los Alamitos, CA: IEEE Computer Society Press.

Sumpter, D. J. T. (2006). The principles of collective animal behaviour. Philosophical Transactions of the Royal Society B - Biological Sciences, 361(1465), 5-22.

Theraulaz, G., \& Bonabeau, E. (1999). A brief history of stigmergy. Artificial Life, 5(2), 97-116.

Theraulaz, G., Bonabeau, E., Sauwens, C., Deneubourg, J.-L., Lioni, A., Libert, F., et al. (2001). Model of droplet dynamics in the Argentine ant Linepithema humile (Mayr). Bulletin of Mathematical Biology, 63(6), 1079-1093.

Trianni, V., Tuci, E., \& Dorigo, M. (2004). Evolving functional self-assembling in a swarm of autonomous robots. In S. 
Schaal et al. (Eds.), Proceedings of the 8th International Conference on Simulation of Adaptive Behavior (pp. 405414). Cambridge, MA: MIT Press.

Tuci, E., Groß, R., Trianni, V., Bonani, M., Mondada, F., \& Dorigo, M. (2006). Cooperation through self-assembling in multi-robot systems. ACM Transactions on Autonomous and Adaptive Systems, 1(2), 115-150.

Vakanas, G., \& Krafft, B. (2001). Coordination of behavioral sequences between individuals during prey capture in a social spider, Anelosimus eximius. Journal of Insect Behavior, 14(6), 777-798.

Vakanas, G., \& Krafft, B. (2004). Regulation of the number of spiders participating in collective prey transport in the social spider Anelosimus eximius (Araneae, Theridiidae). Comptes Rendus Biologies, 327(8), 763-772.

Wang, Z. D., Nakano, E., \& Takahashi, T. (2003). Solving function distribution and behavior design problem for cooperative object handling by multiple mobile robots. IEEE Transactions on Systems, Man, and Cybernetics, Part A: Systems and Humans, 33(5), 537-549.

Ward, C. R., Gobet, F., \& Kendall, G. (2001). Evolving collective behavior in an artificial ecology. Artificial Life, 7(2), 191-209.

West, S. A., Gardner, A., \& Griffin, A. S. (2006). Altruism. Current Biology, 16(13), R482-R483.
Whitesides, G. M., \& Grzybowski, B. (2002). Self-assembly at all scales. Science, 295(5564), 2418-2421.

Wickler, W., \& Seibt, U. (1993). Pedogenetic sociogenesis via the 'sibling-route' and some consequences for Stegodyphus spiders. Ethology, 95, 1-18.

Yamada, S., \& Saito, J. (2001). Adaptive action selection without explicit communication for multirobot box-pushing. IEEE Transactions on Systems, Man, and Cybernetics, Part C: Applications and Reviews, 31(3), 398-404.

Yao, X., Liu, Y., \& Lin, G. (1999). Evolutionary programming made faster. IEEE Transactions on Evolutionary Computation, 3(2), 82-102.

Yim, M., Zhang, Y., \& Duff, D. (2002). Modular robots. IEEE Spectrum, 39(2), 30-34.

Yim, M., Zhang, Y., Roufas, K., Duff, D., \& Eldershaw, C. (2002). Connecting and disconnecting for chain selfreconfiguration with PolyBot. IEEE/ASME Transactions on Mechatronics, 7(4), 442-451.

Zhang, Y., Antonsson, E. K., \& Martinoli, A. (2006). Evolving neural controllers for collective robotic inspection. In A. Abraham, B. de Baets, M. Köppen, \& B. Nickolay (Eds.), Proceedings of the 9th Online World Conference on Soft Computing in Industrial Applications (Vol. 34 of Advances in Soft Computing, pp. 721-733). Berlin: Springer.

Zykov, V., Mytilinaios, E., Adams, B., \& Lipson, H. (2005). Self-reproducing machines. Nature, 435(7039), 163-164.

\section{About the Authors}
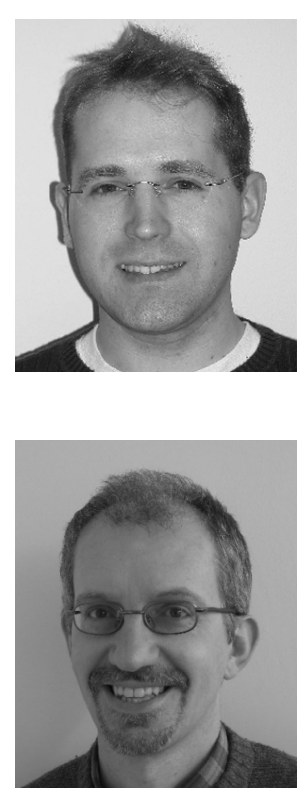

Roderich Groß received a Diploma degree in computer science in 2001 from the University of Dortmund and a Ph.D. degree in engineering sciences in 2007 from the Université Libre de Bruxelles. From 2005-2007, he was JSPS research fellow at the Tokyo Institute of Technology, research assistant at the School of Biological Sciences, University of Bristol, and Marie Curie research fellow at Unilever R\&D Port Sunlight. Currently, he holds a Marie Curie research fellowship at the Ecole Polytechnique Fédérale de Lausanne (EPFL). His research interests include artificial life, computational biology, robotics, and swarm intelligence. $\mathrm{He}$ is member of the program committee of numerous international conferences.

Marco Dorigo is a research director of the F.R.S.-FNRS, the Belgian National Funds for Scientific Research, and of IRIDIA, the artificial intelligence laboratory of the Université Libre de Bruxelles. He is the inventor of the ant colony optimization metaheuristic. His current research interests include swarm intelligence, swarm robotics, and metaheuristics for discrete optimization. Dr. Dorigo is the Editor-in-Chief of the Swarm Intelligence journal. He is an associate editor for IEEE Transactions on Evolutionary Computation, IEEE Transactions on Systems, Man, and Cybernetics, and ACM Transactions on Autonomous and Adaptive Systems, and member of the editorial board of numerous international journals. In 1996 he was awarded the Italian Prize for Artificial Intelligence, in 2003 the Marie Curie Excellence Award, in 2005 the Dr A. De Leeuw-Damry-Bourlart award in applied sciences, and in 2007 the Cajastur International Prize for Soft Computing. Address: IRIDIA, CoDE, Université Libre de Bruxelles, Av. F. Roosevelt, 50 CP 194/6 B-1050 Brussels, Belgium. E-mail: mdorigo@ulb.ac.be 